\title{
Mineração
}

\section{Construção de um modelo computacional para o circuito de ventilação da Mina Esperança}

\author{
Paul Cézanne Pinto \\ Eng. Minas, Mestrando - PPGEM - UFRGS - Porto Alegre, RS, Brasil.E-mail: paul.cezanne@ufrgs.br \\ João Felipe Coimbra Leite Costa \\ Eng. Minas, Professor - PPGEM - UFRGS - Porto Alegre, RS, Brasil.E-mail: jfelipe@ufrgs.br \\ Jair Carlos Koppe \\ Eng. Minas, Professor -- PPGEM - UFRGS - Porto Alegre, RS, Brasil.E-mail: jkoppe@ufrgs.br
}

Paulo Salvadoretti

Eng. Minas, Professor - PPGEM - UFRGS - Porto Alegre, RS, Brasil.E-mail: paulo.saldoretti@ufrgs.br

José Antônio Kurcewicz

Eng. Minas, Mestrando - PPGEM - UFRGS - Porto Alegre, RS, Brasil.E-mail: jantonio@ufrgs.br

Edson Douglas Montedo

\section{Resumo}

A ventilação em uma mina tem como principal objetivo o fornecimento de um fluxo de ar puro a todos os locais de trabalho em subsolo, em quantidades suficientes para assegurar as condições de higiene e segurança dos trabalhadores. A análise da eficiência ou do dimensionamento de um circuito de ventilação pode ser feita por meio de técnicas diretas de análise (algoritmo de Hardy Cross e Leis de Kirchhoff). Essas técnicas são extremamente trabalhosas quando se dispõe de um circuito de muitas malhas. Para uma análise mais detalhada ou previsão de mudanças no circuito de ventilação, empregam-se técnicas de simulação computacional. Essas técnicas foram utilizadas para a construção de um modelo computacional da distribuição do fluxo de ar para a Mina Esperança (SC), tendo como objetivo a adequação do atual circuito de ventilação a NR-22 e o aumento da quantidade de ar fornecida a cada painel em lavra para $2000 \mathrm{~m}^{3} / \mathrm{min}$. Na construção do modelo foram utilizados dados de vazão, diferença de pressão estática, geometria das galerias e as características dos ventiladores. Esses dados, correlacionados pelo simulador, indicaram valores de vazão e pressão compatíveis aos medidos na mina, com diferenças entre os valores simulados e medidos de até $20 \%$, sendo essa diferença adotada, pela equipe de trabalho, para validação dos modelos computacionais. De posse do modelo validado, estudaram-se diversos cenários de lavra futura, antevendo os valores de vazão e pressão a serem encontrados. Portanto as técnicas computacionais para a análise de redes de fluxo comprovaram ser uma importante ferramenta de projeto, diminuindo custos e racionalizando os recursos disponíveis.

Palavras-chave: ventilação, simulação, lavra subterrânea.

Eng. Minas, Carbonífera Metropolitana - Treviso, SC, Brasil

\section{Abstract}

Mine ventilation aims at providing fresh air for all working faces at an adequate flow to assure an appropriate atmosphere to the miners. The design and efficiency analysis in a ventilation circuit can be carried out using Hardy Cross algorithm and Kirchhoff law. In a complex ventilation layout these techniques proved to be extreme laborious. For a more detailed analysis or checking the effect in the ventilation circuit due to mining changes, computational simulation can be used. These simulation methods were used to construct models for Esperança Mine (Brazil). The model construct by simulation aim at checking the adequacy of the ventilation circuit to the NR22 (Brazilian regulation). Also a scenario providing $2000 \mathrm{~m}^{3} / \mathrm{min}$ for each panel was studied. Information necessary to the model include: static pressure, openings dimensions and geometry, and fans operational characteristics. The output from the model includes air flow and pressure along the circuit. Differences between the values predicted by the model and true values are less than 20\%. The model was accepted as valid and therefore used for simulating the ventilation at various mining layouts. Computational ventilation models demonstrate their usefulness in planning and improving underground atmosphere.

Keywords: ventilation, simulation, underground mining 


\section{Introdução}

A ventilação em mina subterrânea tem como principal objetivo fornecer um fluxo de ar fresco (puro), natural ou artificial, a todos os locais de trabalho em subsolo, em quantidades suficientes para manter as condições necessárias de higiene e de segurança dos trabalhadores. Uma ventilação inadequada torna as condições ambientais da mina precárias para os operários e equipamentos, representando para a empresa uma perda de produtividade. De uma maneira simplificada, podemos resumir o papel da ventilação em (Anon., 2000):

- Permitir a manutenção de uma quantidade adequada de oxigênio aos operários.

- Suprimir os gases tóxicos oriundos do desmonte de rochas com explosivos.

- Evitar a formação de misturas explosivas gás-ar.

- Eliminar concentrações de poeiras em suspensão.

- Diluir os gases oriundos da combustão de motores.

- Atenuar a temperatura e a umidade excessiva.

As técnicas computacionais são nos dias atuais, ferramentas de grande utilidade para a análise de redes de ventilação, tendo em vista a grande disponibilidade de aplicativos e da portabilidade dos arquivos e recursos computacionais. A análise direta de circuitos de ventilação é muito limitada, sendo restrita a circuitos parciais dentro do circuito maior de ventilação. É por esse motivo que a análise e otimização dos sistemas de ventilação envolve necessariamente o uso de técnicas computacionais, que facilitam sobremaneira a análise da influência de modificações no circuito de ventilação, reduzindo custos e otimizando os recursos disponíveis (Costa, 1998).

Como em toda técnica de simulação, deve haver preliminarmente uma etapa de coleta de dados para a construção do modelo computacional. Cada circuito de ventilação possui características únicas. Assim, a construção de um banco de dados confiável, que represente as características da mina, é de extrema importância.

Dados sobre as distribuições de vazão e pressão, dos quais obtêm-se o fator de atrito $(\mathrm{k})$ e as resistências equivalentes das galerias, formam a base indispensável para a descrição do comportamento do fluxo de ar. Tais dados devem ser complementados com a medida de outros parâmetros ambientais, tais como teores de gases, poeiras, temperaturas, etc. Com esse banco de dados serão construídas as simulações, bem como a validação de um modelo de fluxo (Clezar, 1999).

Nesse contexto, estabeleceu-se como meta o desenvolvimento de um modelo de simulação do sistema de ventilação da Mina Esperança (SC) (mina de carvão para produção de energia elétrica). Os modelos de simulação pretendem prever a distribuição de fluxo de ar e para tal, é necessário determinarem-se as resistências das galerias que compõem o circuito de ventilação. As etapas do estudo para construção do simulador, aplicação em um caso e sua validação estão descritas no que segue.

\section{Considerações gerais}

As técnicas de ventilação de mina podem ser resumidas basicamente em duas categorias: ventilação natural e ventilação mecânica. A ventilação natural é uma técnica utilizada desde os primórdios da mineração. É causada pela diferença de temperatura do ar no interior da mina em relação ao ar externo.

Com a crescente necessidade de um maior fluxo de ar no interior das minas, desenvolveram-se as técnicas de ventilação mecânica com ventiladores instalados no poço de entrada de ar (insuflação), ou na saída da ventilação (exaustão). Esse desenvolvimento ocorreu, principalmente, a partir da segunda metade do século XIX, com os ventiladores mecânicos de grandes diâmetros, exclusivamente centrífugos e de velocidades reduzidas, movidos por moinhos de vento ou roda hidráulica (Anon., 2000).
Após a primeira guerra, com o grande desenvolvimento da aerodinâmica, foram introduzidos os ventiladores axiais de grande porte, sendo esses hoje em dia os mais empregados. De uma maneira geral, os ventiladores centrífugos são os que melhor se adaptam aos serviços da mina além de serem mais silenciosos. Entretanto os ventiladores axiais são mais baratos, compactos e flexíveis quando ao seu uso, permitindo a regulagem do ângulo de pás de seu rotor, variando os valores de vazão e pressão impostos, sendo, por esses motivos, os mais empregados como ventiladores de poço de ventilação (Montedo, 2002). A análise da eficiência ou dimensionamento de um circuito de ventilação pode ser feita por técnicas diretas, usando as equações de Kirchhoff ou o algoritmo de Hardy Cross para a análise das redes de fluxo (Costa, 1998). Essas técnicas diretas de análise são extremamente trabalhosas, quando temos um circuito composto de muitas malhas (ramos), sendo empregadas apenas em partes do circuito de ventilação (Montedo, 2002). Para uma análise mais detalhada ou previsão de mudanças no circuito de ventilação, empregam-se técnicas de simulação computacional (Costa, 1998). Essas técnicas de simulação se desenvolveram principalmente nos últimos anos, com avanço dos computadores pessoais e da possibilidade de se obterem estimativas sobre possíveis mudanças ou avanços no circuito de ventilação, antevendo-se resultados e economizando-se recursos.

\section{Caracterização do circuito de ventilação da Mina Esperança}

O circuito principal de ventilação da Mina Esperança é composto basicamente pelas galerias de entrada e retorno de ar, pelos divisores de fluxo (tapumes de alvenaria) e pelo exaustor principal. Em seu projeto, a Mina Esperança dispunha de três galerias de entrada de ar e duas de retorno, com 2,3 m de altura por $6 \mathrm{~m}$ de largura, respectivamente. Esse arranjo de circuito não existe na prática. Tanto o circuito de entrada como o de retorno dispõem de um número variável 
Paul Cézanne Pinto et al.

de galerias dependendo do trecho da mina. O controle do número de galerias de entrada e retorno é dificultado pela grande extensão areal da mina e por, eventualmente, ocorrerem caimentos de teto em alguns pontos do circuito. Os elementos do circuito de ventilação, além das galerias, envolvem os divisores de fluxo (tapumes), que são construídos de alvenaria e, posteriormente, rebocados com argamassa, e o exaustor principal (ventilador do tipo axial construído pela Joy Axivane modelo HH-70-50) com 600 cv de potência. $\mathrm{O}$ circuito secundário (dentro dos painéis) é composto pelos ventiladores de frente de lavra, que fornecem $300 \mathrm{~m}^{3} / \mathrm{min}$ de vazão e por tapumes de lona plástica.

No Brasil, as Normas Regulamentadoras do Ministério do Trabalho e Emprego, NR-15 e NR-22, fixam os parâmetros de quantidade e qualidade do ar. Essas normas têm imposto limites cada vez mais rígidos às mineradoras, no sentido de garantir melhores condições de trabalho aos funcionários. A vazão mínima exigida para cada painel em lavra na Mina Esperança, pelas normas vigentes, seria de $1000 \mathrm{~m}^{3} / \mathrm{min}$. No entanto, devido à presença de gases inerentes ao depósito de carvão (metano) e aos produzidos pelo desmonte de rochas com uso de explosivos e poeiras, verificouse que esse valor de vazão é insuficiente para proporcionar conforto e segurança aos trabalhadores. No presente momento, estão em operação na mina três painéis em lavra sendo que a meta da empresa, no que diz respeito à vazão, é o fornecimento de pelo menos $2000 \mathrm{~m}^{3} / \mathrm{min}$ para cada painel. Essa vazão foi considerada pela equipe técnica como suficiente para atender os quesitos necessários ao conforto e segurança dos mineiros.

\section{Metodologia e equipamentos utilizados na construção dos modelos}

Foram coletados dados de vazão, pressão, aspectos geométricos das galerias (largura, altura e comprimento) e parâmetros construtivos dos ventiladores utilizados no circuito de ventilação. Para as medidas de velocidade do ar para o cálculo das vazões, utilizou-se um anemômetro de pás ICEL - Gubintec ${ }^{\circledR}$ modelo AN-10, cuja faixa de operação vai de 0,0 a $45,0 \mathrm{~m} / \mathrm{s}$ com resolução de $0,1 \mathrm{~m} / \mathrm{s}$.

A técnica de levantamento utilizada para a obtenção das velocidades nas seções foi o método dos quadrados (Figura 1), com a qual determina-se o perfil de velocidades na galeria, dividindo-a em seis retângulos imaginários de aproximadamente mesma área. A velocidade é obtida posicionando o anemômetro no centro de cada retângulo e posteriormente realizando a média aritmética das leituras de velocidades.

Para a determinação da perda de pressão estática (perda de carga) em trechos do circuito de ventilação, o manômetro foi conectado a uma mangueira posicionada em um ponto sujeito a uma diferença de pressão estática. Isto é conseguido passando-se a mangueira através de um tapume que separe o circuito de ventilação (ar fresco do ar viciado), de forma que a leitura feita no manômetro represente a diferença de pressão estática do trecho do circuito (Figura 2). Os equipamentos utilizados na coleta de dados de pressão foram: manômetro diferencial Dwyer ${ }^{\circledR}$, modelo 475-0 com escala de zero a $2,45 \mathrm{kPa}$ e menor divisão de escala de 10 Pa e uma mangueira plástica flexível de 12,5 mm de diâmetro.

Com os dados coletados de vazão e pressão, correlacionados pela equação de Atkinson $\left(\Delta \mathrm{P}=\mathrm{R} . \mathrm{Q}^{2}\right)$, onde $\Delta \mathrm{P}$ é a perda de carga, $\mathrm{R}$ é a resistência das galerias e $\mathrm{Q}$ a vazão, pode-se determinar as resistências dos trechos do circuito de ventilação. Para isso, estabeleceram-se pontos de medidas de vazão e pressão,

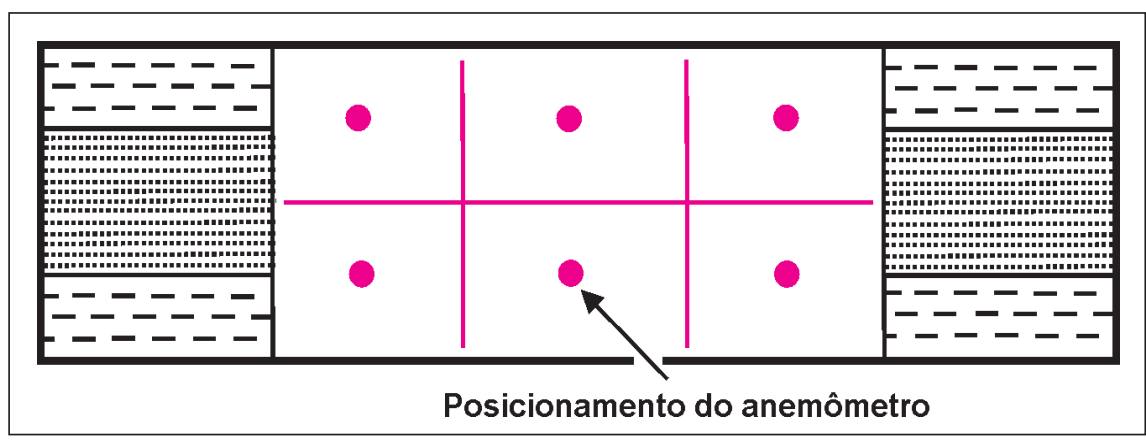

Figura 1 - Levantamento das velocidades com seis posições de medidas.

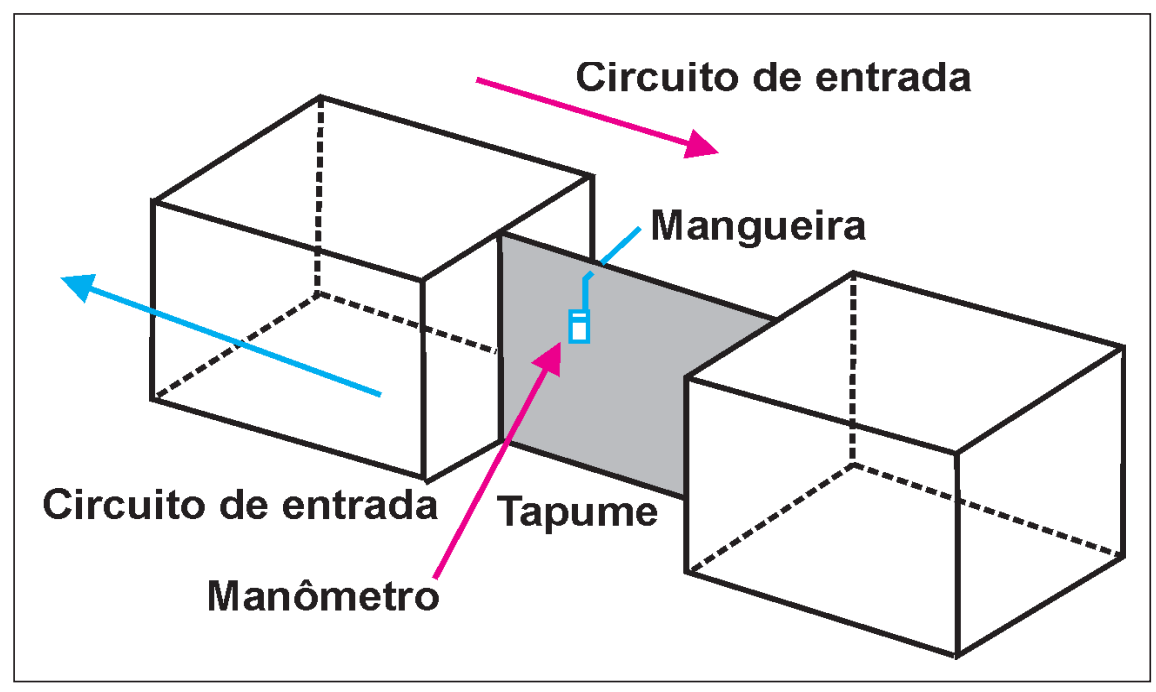

Figura 2 - Esquema para leituras das diferenças de pressão estática. 
dividindo o circuito de ventilação em vários trechos (Figura 3). Nesse tipo de análise, considera-se que a vazão no trecho do circuito é constante (não há fugas) e que as galerias mantêm uma seção regular ao longo do circuito. Como existem medidas de perda de carga no início e fim de cada trecho, basta obterse a diferença desses valores para se ter a perda de carga associada (entrada + retorno). Nessa metodologia, mede-se a perda de carga $(\Delta \mathrm{P})$ associada ao conjunto de galerias pelas quais o ar percorre, ou seja, com essa perda de carga e a vazão medida, determina-se a resistência equivalente do trecho do circuito de ventilação (entrada + retorno). A resistência do circuito de entrada (ou retorno) é obtida dividindo-se por dois o valor encontrado para o trecho do circuito.

O modelo computacional do circuito de ventilação foi alimentado com dados referentes ao período de amostragem, compreendido entre 03/07/02 e 21/ 05/03 (11 campanhas de coleta de dados). Nesse período de coleta, verificouse que o trecho do circuito com os maiores problemas é o corresponde ao encontro dos eixos 13, 17 e 18, parte central da mina. Nesse trecho, ocorre a distribuição do fluxo de vazão, sendo esse o trecho onde os divisores de fluxo (tapumes de alvenaria e portões de curto circuito) apresentam os maiores problemas de conservação, acarretando grande variabilidade nas medidas.

\section{Construção e validação dos modelos computacionais}

Os modelos propostos estão com suas galerias de entrada e retorno de ar em forma resumida, ou seja, o circuito é representado por um diagrama unifilar. Os modelos de circuito de ventilação foram compostos com o intuito de caracterizar o atual arranjo do circuito de ventilação e prever as possíveis distribuições de vazão e pressão nos possíveis cenários de mineração. Outro aspecto levado em consideração foi a adequação do atual circuito à meta da empresa, que é o fornecimento de $2000 \mathrm{~m}^{3} / \mathrm{min}$ para cada frente de lavra.

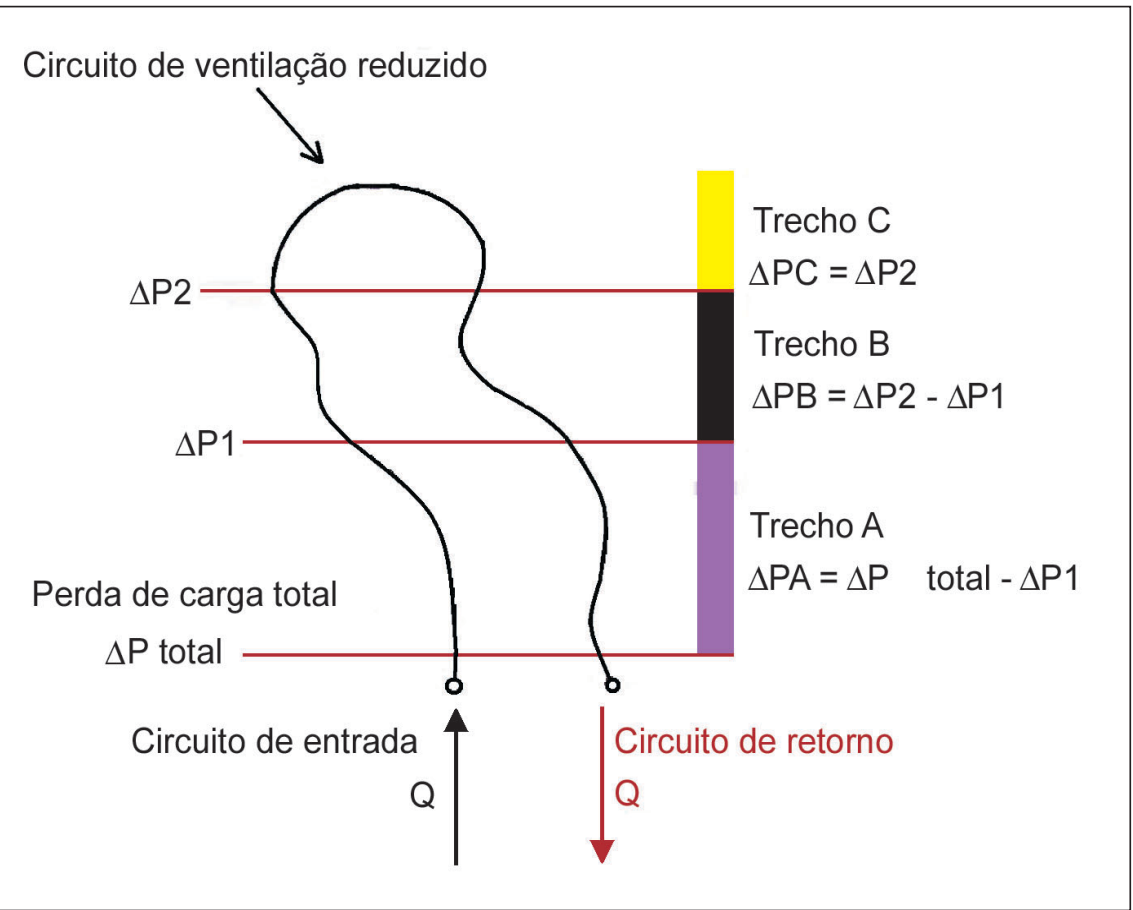

Figura 3 - Esquema para determinação das resistências das galerias.
Na validação do modelo, foram comparados os dados amostrados em 22/05/03 com os dados de saída do aplicativo Ventsim ${ }^{\circledast}$ versão 2,45 (versão educacional). A diferença entre os valores simulados e os valores medidos foi tomada como parâmetro para a consideração do modelo como válido. A equipe de trabalho estabeleceu como $20 \%$ a diferença máxima entre os valores medidos e simulados. Esse valor foi adotado pelo fato de o atual circuito de ventilação possuir trechos com conser- vação inadequada de tapumes, o que dificulta a tomada de medidas de vazão e pressão. Foram comparados os valores de vazão e pressão nos pontos característicos do circuito de ventilação, escolhidos para a coleta de dados, a saber: Poço de exaustão, PV7, PV17, PVCM5, CM5, CM3B, CM3A e PV13R. As Tabelas 1 e 2 apresentam o comparativo entre os valores medidos e simulados de vazão e pressão para os pontos característicos do circuito de ventilação.
Tabela 1 - Comparativo entre os valores de vazão medidos e simulados.

\begin{tabular}{c|c|c|c}
\hline & Valores medidos & Valores simulados & \\
\hline Ponto & Vazão $\left(\mathrm{m}^{3} / \mathrm{min}\right)$ & Vazão $\left(\mathrm{m}^{3} / \mathrm{min}\right)$ & Dif.\% \\
\hline Poço de exaustão & 5152,9 & 5712 & $-9,79$ \\
\hline PV7 Eixo 7 & 4183,7 & 5130 & $-18,45$ \\
\hline PV17 Eixo 17 & 4415,8 & 3048 & 44,88 \\
\hline CM5 & 2473,3 & 2370 & 4,36 \\
\hline CM3B & 1041,8 & 1062 & $-1,90$ \\
\hline CM3A & 1166 & 1146 & 1,75 \\
\hline PVCM5 & 2148,7 & 2370 & $-9,34$ \\
\hline PV 13R & 4984,6 & 4668 & 6,78 \\
\hline
\end{tabular}


Paul Cézanne Pinto et al.

\section{Análise dos resultados}

Analisando os resultados obtidos nos comparativos entre os valores simulados e os valores medidos, verificase que o modelo proposto satisfaz as condições exigidas para todos os pontos de controle, com exceção dos pontos situados no eixo 17 (PV17, para os valores de vazão e PVCM5 para os valores de pressão). Esse trecho do circuito de ventilação é o que apresenta maiores problemas na conservação de seus divisores de fluxo. Esse fato dificulta a tomada de medidas de pressão e vazão, resultando em valores inadequados de entrada e fora do limite estabelecido para validação. Como o modelo satisfaz as condições impostas para todos os outros trechos do circuito de ventilação, esse foi considerado como validado. A partir do modelo de fluxo validado, construiu-se um modelo adequando o atual circuito de ventilação às metas da empresa, ou seja, fornecimento de $2000 \mathrm{~m}^{3} / \mathrm{min}$ por painel. Para isso, utilizaram-se apenas os recursos disponíveis na mina. O booster utilizado no modelo tem características semelhantes ao disponível e as fugas foram diminuídas. Os resultados obtidos evidenciaram que os ventiladores disponíveis na mina não são capazes de fornecer a vazão planejada para os painéis em lavra. Portanto é necessário um ajuste na atual configuração de pás dos mesmos para um fornecimento de uma maior vazão ou a aquisição de outros ventiladores que atendam essa premissa.

Outro fato constatado é a necessidade de uma melhora na qualidade de alguns tapumes da mina. Para que seja atendida a meta, deve-se fazer uma reforma nos tapumes de alvenaria, diminuindo as perdas e evitando-se a recirculação de ar. Apesar desses fatos, fica evidente, na análise da Tabela 3, que é possível uma melhora considerável no circuito de ventilação. A disposição dos painéis em paralelo, além de atender as normas vigentes, melhora as condições de trabalho dos operários. Outro fato positivo é o da viabilidade de implemen-

Tabela 2 - Comparativo entre os valores de pressão medidos e simulados.

\begin{tabular}{c|c|c|c}
\hline & Valores medidos & Valores simulados & \\
\hline Ponto & Pressão (Pa) & Pressão (Pa) & Dif.\% \\
\hline Poço de exaustão & 1860 & 1862 & $-0,11$ \\
\hline PV7 Eixo 7 & 980 & 844 & 16,11 \\
\hline PVCM5 & 100 & 259 & $-61,39$ \\
\hline PV 13R & 450 & 396 & 13,64 \\
\hline
\end{tabular}

Tabela 3 - Resultados obtidos com a simulação para os valores de vazão considerando o modelo de circuito proposto.

\begin{tabular}{c|c|c|c}
\hline & Valores medidos & Valores simulados & $\begin{array}{c}\text { Dif. do simulado e o } \\
\text { pretendido } \\
\left(\mathbf{2 0 0 0} \mathbf{~ m}^{3} / \mathbf{m i n}\right)\end{array}$ \\
\hline Ponto & Vazão $\left(\mathbf{m}^{3} / \mathrm{min}\right)$ & Vazão $\left(\mathbf{m}^{3} / \mathrm{min}\right)$ & $\%$ \\
\hline CM5 & 2473,3 & 1914 & $-4,3$ \\
\hline CM3A & 1166 & 2004 & 0,2 \\
\hline CM3B & 1041,8 & 1608 & $-19,6$ \\
\hline
\end{tabular}

tação dessas mudanças, visto que foram utilizados apenas os recursos disponíveis na mina.

O resultado mais importante obtido com o modelo de fluxo para o circuito de ventilação em paralelo é que, apesar de as vazões em dois painéis serem inferiores ao estabelecido como meta, tais vazões ficaram bem próximas a $2000 \mathrm{~m}^{3} /$ min. O modelo estabelecido serve como uma primeira etapa para se atingir o estabelecido. A Figura 4 apresenta o modelo de fluxo para o circuito de ventilação proposto com os respectivos valores de vazão.

\section{Conclusões}

Com os resultados obtidos do modelo computacional proposto, pode-se verificar que o modelo validado para o circuito de ventilação da Mina Esperança apresentou resultados compatíveis. O modelo retornou valores dentro de faixas aceitáveis de erro nos trechos do circuito de ventilação, onde se tem a possibilidade de coletar dados de vazão e pressão de forma adequada, com diferenças entre o que é medido e simulado na ordem de $20 \%$. $\mathrm{O}$ trecho em que o modelo retornou valores com diferenças maiores é onde ocorrem os maiores problemas na determinação das medidas. Portanto, para se obterem melhores resultados nesses pontos, deve-se primeiramente melhorar as condições do circuito de ventilação, no que se refere à qualidade dos tapumes e portões de curto-circuito.

Os modelos de simulação possibilitam a previsão do desempenho do circuito de ventilação, no que se refere às necessidades de vazão para os futuros layouts do circuito e, conseqüentemente, para melhorias na atmosfera da mina. Essa metodologia de trabalho mostrou-se eficaz e pode ser estendida a outras minas, ganhando importância à medida que a ventilação representa uma parcela significativa dos custos de produção. Em adição, melhorias no projeto e na sua execução elevam a qualidade do ambiente de trabalho, o conforto e a produtividade dos operários. 


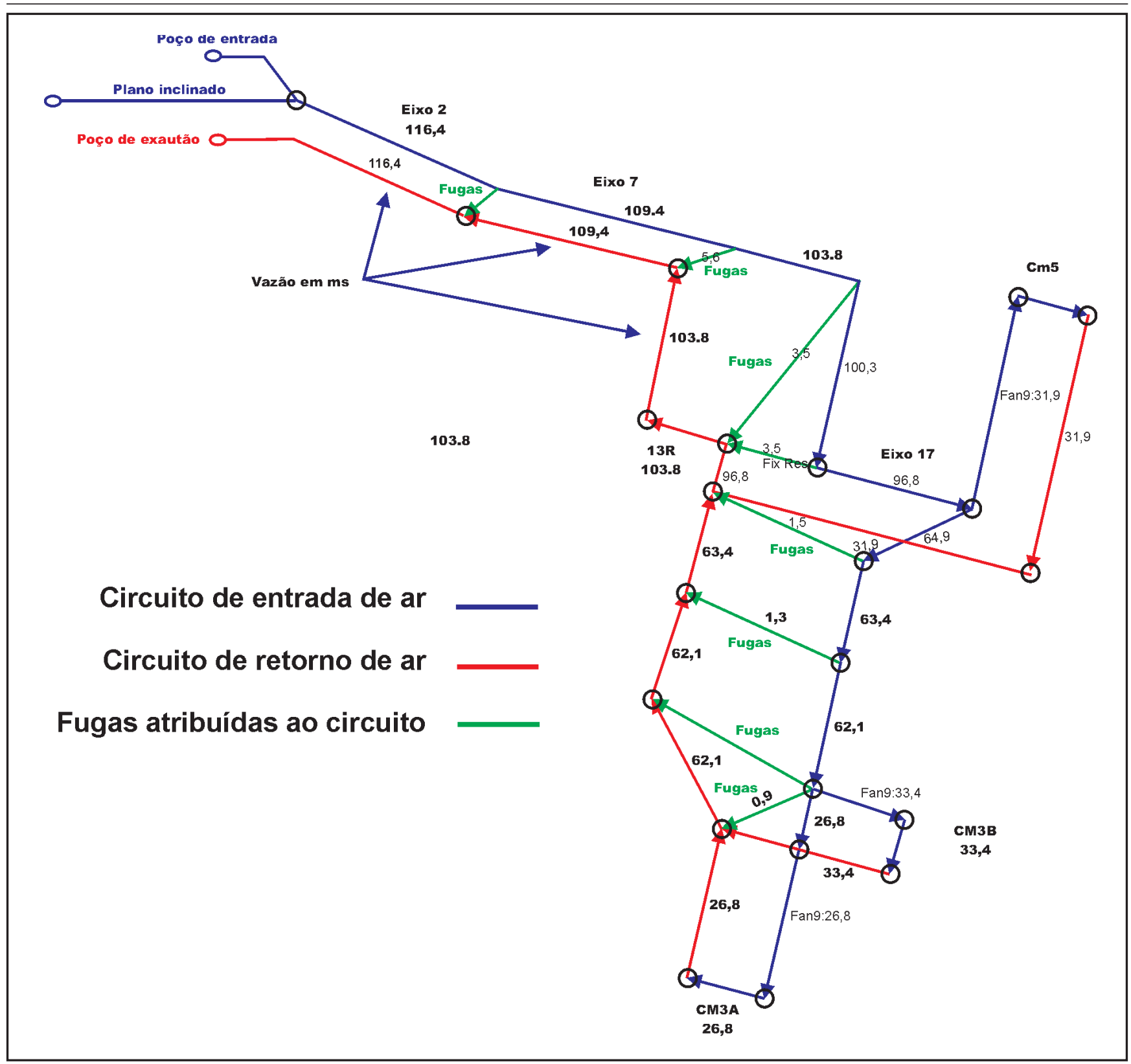

Figura 4 - Modelo de fluxo para o circuito de ventilação proposto.

\section{Agradecimentos}

Os autores agradecem à Carbonífera Metropolitana S.A., ao CNPq e à Finep (CTENERGIA).

\section{Referências bibliográficas}

ANON., CIA MINEIRA DE METAIS, Ventilação, manual de procedimentos $e$ dimensionamento de ventilação, Vazante-MG: 2000. 62p. (Artigo interno da empresa).

CLEZAR, C. A., NOGUEIRA, A. C. R. Ventilação industrial. Florianópolis: Ed. da UFSC, 1999. 298p.

COSTA, J. C. A. Análise de redes de ventilação - estudo de caso mina Medrado-BA,

Campina Grande: 1998. 80p. (Dissertação de Mestrado).
NR-22, Segurança e Saúde Ocupacional na Mineração (122.000-4), Brasil: Ministério do Trabalho e da Previdência Social Secretaria Nacional do Trabalho, 2002.

MONTEDO, E. D. Ventilação de mina abordagem teórica. Relatório Técnico da Carbonífera Metropolitana, CriciúmaSC: 2002. 244p. (Inédito).

Artigo recebido em 11/07/2003 e aprovado em 24/11/2003. 